\title{
RESTRUCTURACIÓN NEOLIBERAL, REGIONALIZACIÓN Y DESIGUALDAD EDUCATIVA EN LA EDUCACIÓN PRIMARIA DEL URUGUAY DE LOS NOVENTA
}

\author{
Tabaré Fernández Aguerre*, Santiago Cardozo Politti**, \\ Mahira González Bruzzesse ${ }^{* * *} \mathrm{y}$ \\ Cecilia Rodríguez Ingold****
}

\section{INTRODUCCIÓN}

El objetivo de este artículo es dimensionar los efectos que habrían tenido las estructuras de oportunidad territorialmente diferenciadas sobre los logros educativos de niños en edad escolar, en el

* Licenciado en Sociología, Facultad de Ciencias Sociales, Universidad de la República, Uruguay; Magister en Ciencias Sociales, Universidad Alberto Hurtado, Chile; Doctor en Ciencia Social con especialidad en Sociología, El Colegio de México. Premio Academia Mexicana de Ciencias 2005 Tesis de Doctorado. Consultor Instituto Nacional de Evaluación Educativa, México (2002-2005). Ex Coordinador Nacional de PISA (2007); Presidente de la Comisión Coordinadora del Interior, Universidad de la República (20142017). Profesor Agregado en Régimen de Dedicación Total, Departamento de Sociología, Facultad de Ciencias Sociales, Universidad de la República.

** Licenciado, Magister y Doctor en Sociología, Facultad de Ciencias Sociales, Universidad de la República, Uruguay. Especialista en Evaluación en Educación. Investigador Principal, Dirección de Investigación, Evaluación y Estadística (DIEE) de la Administración Nacional de Educación Pública. Profesor Adjunto, Departamento de Sociología, Facultad de Ciencias Sociales, Universidad de la República.

*** Licenciada en Sociología, Facultad de Ciencias Sociales, Universidad de la República, Uruguay y Magister en Gestión Educativa, Universidad ORT, Uruguay. Profesora Asistente del Núcleo de Estudios Interdisciplinarios sobre Sociedad, Educación y Lenguaje en Frontera (NEISELF), Centro Universitario de Rivera, Universidad de la República.

**** Licenciada en Economía del Desarrollo, Universität Bayeruth, Alemania. Profesora Ayudante del Departamento de Economía, Facultad de Ciencias Sociales, Universidad de la República. 
contexto en que Uruguay instauraba explícita y sistemáticamente el modelo neoliberal a comienzos de los años noventa.

Este es un análisis histórico y territorial de los logros educativos, que pretende aportar en aspectos escasamente estudiados antes en Uruguay. El enfoque predominante para el análisis de la desigualdad educativa ha mostrado el papel crucial del capital cultural y económico de las familias, la magnitud y factores con que aparece el "efecto escuela" y el sesgo de selección que yace debajo de la visible segmentación institucional público/privado que caracteriza al país (Fernández, 2002; Fernández, 2003). Estos hallazgos han sido reportados desde la década del sesenta por el Informe de la Comisión de Inversiones y Desarrollo del Estado (CIDE, 1965) y a principios de los noventa por la CEPAL (CEPAL, 1991; Rama, 1991). Nuestro propósito aquí es innovar incorporando la consideración sobre los efectos del territorio, postulando tres niveles de análisis (regiones, departamentos y áreas locales). Es preciso destacar que esas estructuras de oportunidades con elementos de cada nivel territorial resultan del efecto acumulado de varios macroprocesos históricos de "larga duración", tanto económicos, políticos, culturales y demográficos.

Nuestro interés analítico se concentra en una generación nacida a mediados de los ochenta y que concluye su escolarización a mediados de los noventa, esto es, desde el final de la Dictadura Militar (19731984) y la entrada en vigencia del Tratado de Asunción, constitutivo del Mercado Común del Sur (MERCOSUR) (1995). Durante este tiempo período se produce la explícita sustitución del modelo económico y social que el país había configurado, tanto sectorial como territorialmente, desde por lo menos 1911, siendo progresivamente sustituido por otro modelo, neoliberal ${ }^{1}$, que mostraría sus impactos a fines de los noventa y comienzos de los años dos mil. Los datos censales provistos por la Evaluación Nacional de Aprendizajes en los sextos años de Primaria de 1996 configuran una matriz de información valiosa para describir la dimensión territorial de la desigualdad educativa, tomando como referencia la configuración de regiones económicas, departamentos y estructuras de oportunidad locales que hemos identificado hacia fines de los años setenta en otros trabajos (Fernández \& Ríos, 2013; Fernández, 2018).

Dos son las preguntas que guían este trabajo: ¿acceden los alumnos a punto de egresar de Primaria a los mismos conocimientos básicos en Matemática y Lectura independientemente de su lugar de

1 Si bien ninguno de los actores gubernamentales clave en los sucesivos gobiernos aceptó la adscripción neoliberal para esta nueva matriz, resulta una descripción extendida y aceptada por los distintos cientistas sociales. En este sentido será usada aquí. 
residencia? En segundo lugar, ¿ existen variaciones territoriales en los logros sucesivos de la trayectoria escolar debidos al territorio?

Nuestra hipótesis es que tales diferencias territoriales constituyeron elementos fundamentales de la estructura de desigualdad de oportunidades educativas que enfrentaban los escolares entre 1990 y 1996 y como tales, configuran determinantes de los logros en la trayectoria escolar, tanto en el acceso a la Educación Inicial como al cabo de la Primaria en las áreas centrales de Matemática y Lectura. Esta estructura territorial desigual habría operado modificando el "piso local" de oportunidades desde el que partió la socialización familiar, la socialización escolar temprana y la transmisión intergeneracional de capital cultural.

En la primera parte del artículo, desarrollamos en forma teórica el concepto de espacialización social, proceso que resulta clave para comprender las estructuras de oportunidades que creemos se han diferenciado territorialmente en el país. Luego presentamos brevemente la configuración regional del Uruguay a fines del siglo XX, en la que se basa nuestra hipótesis. En tercer lugar, presentamos las fuentes de datos a utilizar y el método, para luego discutir hipótesis y presentar resultados.

\section{EL TERRITORIO Y LA ESPACIALIZACIÓN SOCIAL}

El concepto de territorio que aquí utilizamos excede una noción restrictiva, determinante, la que podría atribuírsele a la geografía física; y también deja de lado la idea de "territorio-escenario", propia del interaccionismo simbólico.

Entendemos que las acciones sociales distribuyen (en forma intencional o como externalidad) geográficamente recursos, instituciones y poblaciones. Algunos geógrafos, sociólogos y urbanistas han denominado este fenómeno como espacialización social (Paasi, 1986; Shields, 1991). Analíticamente, estas espacializaciones pueden ser agrupadas en procesos, lo cual permite observar la configuración de estructuras (Giddens, 1992; Sewell W. J., 2006). Cuando el interés analítico se fija en períodos largos de tiempo, la "longue durée" de la cual hablaba Braudel (1958), es posible identificar macroprocesos de espacialización social que conllevaron a distintas configuraciones territoriales, cada una con cierta institucionalidad e identidad (Paasi, 1986).

En estudios previos (Fernández, 2002; Fernández, 2018), hemos descrito estos macroprocesos, de los cuales interesa señalar aquellos que están a la base de la configuración territorial observable a fines de los años ochenta y que resultan pertinentes a los efectos de este estudio: (i) la departamentalización, ii) la agriculturización; (iii) la industrialización; (iv) la balnearización; y (v) la micro-urbanización. Estos macroprocesos, contribuyeron tanto a reproducir como a recrear las diferencias territoriales heredadas desde la geopolítica y distribución de tierras coloniales. 
El Estado fue un actor predominante en la diferenciación territorial, a través de políticas sectoriales de incentivos, por ejemplo, al ferrocarril, a la industria o a la vitivinicultura; en la política comercial proteccionista de la producción agrícola nacional; como ordenador del territorio (aunque varias veces más por omisión que por acción); fundando ciudades o directamente interviniendo como inversor. A lo largo de estos doscientos cincuenta años, los territorios no solo se diferencian progresivamente unos de otros (procesos de diferenciación horizontal), sino que también se generan relaciones de complementación (o conflictos), dependencia y subordinación, estableciéndose una diferenciación vertical, estratificada entre los territorios. Aquellos macroprocesos integraron territorios a institucionalidades o cadenas productivas, a la vez que diferenciaron territorios por su origen poblacional o sus recursos institucionales. Al cabo de doscientos años, a las grandes macroregiones coloniales de base cultural y económica, se agregaron los "departamentos" como territorios fuertemente institucionalizados desde la política, y las áreas locales, muy débiles en la institucionalidad política, con grandes diferencias en los recursos. Esta idea de que la estructura de oportunidades estaba territorialmente estratificada fue descripta punto por punto por primera vez en la Sociología por el informe de CLAEH/CINAM (1963).

Podría avanzarse aún más sosteniendo que las características que tuvieron estos macroprocesos también determinan características en las estructuras de oportunidades, según fueran satisfactores provistos desde el Estado o desde la actividad económica (mercado). La primera dimensión generó una diferenciación vertical de las estructuras locales de oportunidad. La provisión de servicios públicos a nivel territorial fue resultado del proceso de departamentalización del territorio: esto es una construcción político-institucional que dio lugar a redes urbanas muy diferentes en cada departamento según la existencia e importancia previa de las localidades fundadas hasta $1830^{2}$. Políticamente hubo prestaciones de política social reservados a las capitales departamentales, que sólo en los pocos departamentos policéntricos se observan tempranamente en otras ciudades. A su vez, la subdivisión territorial del departamento fue efecto exclusivo de la impartición de Justicia (los juzgados de paz) y de policía, creándose las secciones.

Cada organismo público creó sus regiones administrativas, algunas coincidentes con todo el departamento, y otros con aspectos instrumentales propios de sus tareas (como el Ministerio de Obras Públicas o la empresa eléctrica). Con excepción de tres juntas

2 Por ejemplo, departamentos policéntricos como Colonia, Canelones, Soriano y Maldonado, así como monocéntricos como Salto, Paysandú, Durazno, Lavalleja. 
locales en todo el territorio nacional, no hubo en la historia nacional una institucionalidad política territorial subdepartamental ${ }^{3}$.

La segunda dimensión se manifestó en macroprocesos mencionados en la sección anterior: la inmigración, la agriculturización, la balnearización y la industrialización. Finalmente, deben contemplarse dos procesos poblacionales específicos, que, si bien están fuertemente asociados a los anteriores, destacan por su especificidad. Por un lado, la "micro-urbanización" resultante de la modernización rural y de la agriculturización, desarrollado casi por completo fuera de la planificación y sin regulación efectiva en los aspectos del ordenamiento territorial (Alvarez Lenzi, 1986), con alcances que variaron según el tipo de departamentalización alcanzada en el siglo XIX (Fernández et al., 2018). Por otro lado, los movimientos migratorios, tanto de recepción como de expulsión, fueron redefiniendo aportes culturales, lingüísticos y tecnológicos muy diferenciados en cada territorio.

\section{ESTRUCTURA DE OPORTUNIDADES Y ÁREAS LOCALES}

El término "estructura de oportunidad", que aquí utilizamos como conceptualización central para describir un área local, está inspirado en el enfoque propuesto a fines de los años noventa por Carlos $\mathrm{H}$. Filgueira y Ruben Kaztman (1999). Originalmente, aquella se entendía como las probabilidades de acceso a bienes, servicios o a actividades que inciden sobre el bienestar del hogar porque le facilitan el uso de recursos propios o le suministran recursos nuevos.

Los bienes y servicios que componen la estructura de oportunidades se presentan con diferentes calidades, variedades y costos, dependiendo entre otras cosas, de quién sea el proveedor: los mercados (por ejemplo, para alimentos, electrodomésticos, recreación y vestimentas), el Estado o la comunidad, más allá del propio hogar (el vecindario, las asociaciones, la familia más extensa, etc.). Esta distinción tripartita, originaria entre otros de Gøsta Esping-Andersen (1990), ha sido fuertemente criticada, en particular por las feministas, porque ignora la producción de bienestar que se realiza en el hogar, en particular a cargo de la mujer (Aguirre, 2009). A los efectos del análisis territorial que aquí queremos hacer, resulta imprescindible deslindar la esfera doméstica de estas estructuras, sin por esto desconocer que posiblemente el mayor peso explicativo en los logros durante la infancia radique en las prácticas de alimentación, prevención sanitaria, crianza y estimulación cognitiva que el hogar realiza (Tuñón y Poy, 2016; Bernstein, 1971).

3 Recién en 2009 se aprueba la Ley de Descentralización creando los "municipios" en todas aquellas localidades mayores a 1000 habitantes. 
Hasta este punto, nuestra noción no se distingue gruesamente de la trabajada por Kaztman y Filgueira (1999). Sin embargo, desde el punto de vista territorial resulta claro que los individuos no se encuentran ubicados geográficamente a una misma distancia de la provisión de esos bienes o del uso de servicios. ¿Cuánto puede suponerse que un individuo se desplace en condiciones cotidianas, diarias, a los efectos de realizar las compras necesarias para la preparación de los alimentos, para llegar a su lugar de trabajo, para realizar controles de salud a los niños, cobrar su jubilación o asistir a la Escuela Primaria?

Esta pregunta práctica está en la base de la introducción de los términos "localidad centro de servicios" y "áreas locales" hecha por CLAEH/ CINAM (1963). Primero georreferenciaron la ubicación de los servicios públicos correspondientes a las dimensiones de satisfacción de las necesidades humanas indicadas más arriba. Esto les permitió observar que se conformaban "clusters de servicios" localizados físicamente en torno a centros poblados, cuya dimensión, calidad y heterogeneidad correlacionaban (aunque no fuertemente) con el número de pobladores residentes desde los parajes hasta las capitales. Sobre este primer hallazgo formularon una tipología (sería más correcto llamarle estratificación) que clasificó 257 centros poblados de inicios de los años sesenta.

\section{CONFIGURACIÓN REGIONAL DE URUGUAY EN EL ÚLTIMO CUARTO DEL SIGLO XX}

Un estudio previo (Fernández, 2018) presentó evidencia de una configuración regional muy particular en el territorio del Uruguay entre fines de los años cincuenta y hasta fines de la década de 1970. Esta configuración territorial es precisamente en la que nace y se socializa la generación de alumnos de Primaria que son el universo donde testearemos nuestras hipótesis. Resaltamos aquí cómo estas regiones habrían operado sobre la conformación de expectativas laborales y sobre la demanda educativa (Checchi, 2006) en relación con las nuevas generaciones, con base en algunas primeras hipótesis que expusimos en otro trabajo anterior (Fernández, Rodríguez y González, 2018).

En el presente trabajo y con el objetivo de hacer más evidente la tesis anterior, dejaremos de lado las particularidades observadas en el departamento de Montevideo, y nos concentraremos en siete regiones del interior de la República: i) el Gran Canelones; ii) el Suroeste; iii) el Litoral; iv) la Frontera Noreste; v) la cuenca de la Laguna Merin; vi) el Este; y vii) el Centro ${ }^{4}$.

4 Esta decisión teórica y metodológica sigue la tradición que ha dejado instalada Veiga $(1991 ; 2010 ; 2015)$. 
La región del Gran Canelones incluye todo el departamento homónimo junto con pequeñas áreas locales de los departamentos de Maldonado y Lavalleja. En 1985 concentraba el 12.7\% de la población total del país y contaba con el $21 \%(\mathrm{n}=135)$ de las localidades censadas. Está conformada sobre la base de las zonas agroeconómicas dedicadas a la hortifruticultura intensiva (CLAEH/CINAM, 1963). Es una región caracterizada por la más densa red de pequeñas localidades, con baja predominancia de la capital y muchas ciudades intermedias que son centros de servicios para áreas locales importantes, todas ellas muy interconectadas por carreteras y caminos departamentales, que además la unen radialmente a la capital del país, Montevideo. La matriz productiva está muy diversificada con cadenas agroindustriales intraregionales (la remolacha azucarera, avícolas, carne porcina y la elaboración de vinos) y extraregionales (frigoríficos, fábricas de calzado), así como producción hortifrutícola y granjera para el consumo. La proximidad e integración vial con Montevideo amplía los mercados, incluido el laboral, a la máxima escala que puede encontrarse en el país, sea por la presencia de sectores muy dinámicos y con alta rentabilidad de capital humano, sea por la presencia de sectores masivos (como el comercio, la construcción y los servicios) que no requieren alto capital humano. Aunque grandes, no son necesariamente mercados perfectos, entre otras razones, debido a los circuitos comerciales en varios aspectos distorsionados tal como lo expusiera el informe CLAEH/CINAM (1963).

El Sur-Suroeste está conformado por algunas áreas locales del departamento de Florida, y los departamentos de San José y de Colonia, correspondiéndose en sentido amplio, con las áreas agroeconómicas que combinan la fruticultura, la agricultura de forrajes y la lechería (conforme a la regionalización CLAEH /CINAM, 1963). En 1985 concentraba el $8 \%$ de la población total del país y contaba con el 15\% $(\mathrm{n}=94)$ de las localidades censadas. Es una extensa región de base agrícola con inversiones en ganadería intensiva: fruti y vitivinicultura, campos de recría, campos de engorde para la industria frigorífica alojada predominantemente en Montevideo, algunas industrias sustitutivas importantes en la rama textil y papelera; pero con predominancia de la cadena agroindustrial de la lechería. Esta región se benefició por un cambio en el modelo económico impulsado desde mediados de los años setenta: la quita al monopolio de la empresa láctea paraestatal CONAPROLE en el abastecimiento de leche y derivados industriales al mercado de Montevideo. Esto permitió el florecimiento de pequeñas y medianas industrias lecheras, tanto familiares como cooperativas y sociedades anónimas, y la consiguiente demanda por una fuerza 
de trabajo con un nivel de formación post-primario, calificada. En relación a la capital del país y su mercado de trabajo, los incentivos descriptivos para la región del Gran Canelones podrían aplicar aquí, con dos excepciones. En primer lugar, es de tomar en cuenta que las distancias mayores entre la capital y la región generan una discontinuidad geográfica al punto que resulta altamente costoso residir en la región y desplazarse diariamente a trabajar en el departamento de Montevideo. En segundo lugar, la demanda de trabajo típica local en sectores dinámicos podría pensarse que hace rentable la permanencia en el territorio, la acumulación de capital humano y la inserción semi o directamente calificada en empresas locales, en lugar de la emigración. Esto conllevaría a observar menor heterogeneidad en los logros educativos.

La Región Central del país se conformó sobre la vieja zona "entre los ríos Yi y Negro", actual departamento de Durazno, todo el departamento de Flores, el noroeste de Florida, el este de Soriano y el sur de Tacuarembó. En 1985 concentraba el 6,1\% de la población total del país y contaba con el $18 \%(n=115)$ de las localidades censadas. No alcanzó más que una baja diversificación sectorial, de base ganadera aunque con predominio vacuno, especializada en las actividades de mejoramiento de razas y de invernada y con escasa presencia industrial. De las siete, es la segunda con mayor cantidad de localidades y la más alta proporción (89\%) de micro-urbanizaciones (parajes y localidades).

El Litoral comprende una amplia franja regada por el río Uruguay, incluyendo buena parte de los departamentos de Soriano, Río Negro, Paysandú, Salto y Artigas. En 1985 concentraba el 12,9\% de la población total del país y contaba con el 15\% $(\mathrm{n}=98)$ de las localidades censadas. Su rasgo más nítido es que las dos ciudades más grandes del país luego de Montevideo están en esta región: el Censo de 1985 indicó que la población total de Salto alcanzaba los 80.823 habitantes y Paysandú contó con 68.468 habitantes. En los ochenta conformaba una extensa región asentada en un importante grado de diversificación sectorial económica: agricultura cerealera, citricultura, horticultura de contra-estación, caña de azúcar junto con una ganadería tempranamente modernizada y distintos sectores industriales (textil, cueros, frigoríficos, alimentación, cementos).

La frontera Noreste, sobre la frontera con Brasil, desde Artigas hasta Rio Branco y Tacuarembó, concentraba en 1985 el 9,3\% de la población total del país y contaba con el $16 \%(\mathrm{n}=101)$ de las localidades censadas. En esta región también la micro-urbanización alcanzó los niveles más altos: el $85 \%$ de las urbanizaciones eran 
parajes o localidades, pero reunían el 6\% de la población. No alcanzó más que una muy baja diversificación sectorial sobre una base de ganadería extensiva (vacuna y ovina), horticultura y papas (en chacras próximas a Rivera, Tacuarembó y Melo), con ensayos limitados en arroz y cítricos. Solo hubo industrias ligeras, ligadas a la alimentación y la higiene. Es una región históricamente caracterizada por sus interacciones comerciales y culturales con los municipios limítrofes del Estado de Rio Grande do Sul (Mazzei y De Souza, 2013). En 1910, Rivera era la estación del ferrocarril con el mayor volumen de tráfico luego de Montevideo (Travieso, 2017) y la construcción del puente caminero y ferroviario "Barón de Mauá" en 1927, todo un símbolo para su época, marcó la intensidad e importancia del intercambio.

Tabla 1. Distribución de la población y de las localidades en las regiones. Uruguay 1985

\begin{tabular}{|c|c|c|c|c|c|c|}
\hline & Población total & $\%$ en pais & Num. Loc & Pob. Urbana & $\%$ Urb. País & \% Urb. Reg \\
\hline Montevideo & $1,311,976$ & $44.4 \%$ & 4 & $1,255,106$ & $48.6 \%$ & $95.7 \%$ \\
\hline Gran Canelones & 374,090 & $12.7 \%$ & 135 & 302,770 & $11.7 \%$ & $80.9 \%$ \\
\hline Sur-Suroeste & 221,317 & $7.5 \%$ & 94 & 168,239 & $6.5 \%$ & $76.0 \%$ \\
\hline Litoral & 380,867 & $12.9 \%$ & 98 & 321,186 & $12.4 \%$ & $84.3 \%$ \\
\hline Frontera & 273,363 & $9.3 \%$ & 101 & 218,074 & $8.4 \%$ & $79.8 \%$ \\
\hline Laguna Merin & 67,408 & $2.3 \%$ & 28 & 52,568 & $2.0 \%$ & $78.0 \%$ \\
\hline Este & 146,104 & $4.9 \%$ & 61 & 130,269 & $5.0 \%$ & $89.2 \%$ \\
\hline Central & 180,116 & $6.1 \%$ & 115 & 132,875 & $5.1 \%$ & $73.8 \%$ \\
\hline
\end{tabular}

Fuente: elaboración propia con base en microdatos del Censo de Población de 1985, INE.

Dentro de la vieja región colonial de la frontera con el Brasil, y en torno a la cuenca de la Laguna Merin, desde 1940 creció una creciente actividad arrocera, en su fase agraria como industrial que, si bien estuvo combinada con la ganadería extensiva propia de la zona, rearticuló una amplia zona interdepartamental de Treinta y Tres, la tercera sección judicial de Cerro Largo (Río Branco) y el norte del Departamento de Lavalleja (secciones judiciales $9^{\mathrm{a}}$ y $10^{\mathrm{a}}$ ). El arroz creó nuevos vínculos con proveedores, productores y compradores del Brasil (por el origen de los capitales, de los propietarios y muchas veces también de la fuerza de trabajo).

El Este, Maldonado y Rocha, fueron progresivamente reconfigurados torno a la creciente importancia del turismo tanto en la inversión en activos como en la demanda de empleo. 

el Uruguay (1960-1990).

Fuente: elaboración del Mag. Victor Borrás con base en microdatos del Proyecto "Territorios y Desigualdades". Las subdivisiones menores refieren a las secciones censales en que se divide el país a 2011.

\section{ESTRUCTURA LOCAL DE OPORTUNIDADES EDUCATIVAS}

Consideramos en este trabajo las dimensiones educativas identificadas por CLAEH/CINAM (1963): la Enseñanza Primaria, el primer ciclo de la Enseñanza Media (hasta 1986, solo provista como Secundaria General) y la Enseñanza Técnica. Agregamos, además, la provisión de Educación Inicial, en razón de que existe suficiente evidencia en Uruguay para mostrar su incidencia determinante en la adaptación escolar, el aprendizaje de la lecto-escritura, la repetición y, finalmente, el aprendizaje en las áreas instrumentales de la Lengua y la Matemática al cabo de la Primaria, precisamente para la cohorte que estudiaremos (Ravela et al., 2000). La provisión educativa que el Estado reconoce y acredita, está provista principalmente por la Administración Nacional de Educación Pública (ANEP) a través del Consejo de Educación Inicial y Primaria (CEIP) y del Consejo de Educación Secundaria; el Plan de Centros de Atención a la Infancia y la Familia (CAIF), centros privados de educación inicial reconocidos por el Ministerio de Educación, y colegios primarios privados habilitados por el CEIP.

La Educación Pública en Uruguay está organizada institucionalmente con base en la departamentalización. En cada departamento desde fines del siglo XIX, hay un Inspector Departamental como máxima autoridad en el territorio con la función de fiscalizar 
administrativamente, orientar pedagógicamente, evaluar resultados y asignar recursos. La formación docente está a cargo de un Instituto Normal departamental ${ }^{5}$, la Educación Secundaria desde la Ley de 1912 se organiza a nivel departamental y la provisión de cargos docentes se realiza también a este nivel territorial.

Existe consenso en la bibliografía al señalar que la estructura institucional para la provisión de enseñanza Primaria explica los logros alcanzados. El nivel de Primaria se ha considerado universalizado desde 1940. Por el contrario, la estructura de provisión del servicio de Enseñanza Secundaria, solo departamentalizada, explica la lentitud y la baja cobertura alcanzada. Para inicios de los sesenta, entre los 13 y hasta los 17, edades coincidentes normativamente con la Educación Secundaria, se observaban caídas fuertes en la cobertura: desde el $83 \%$ hasta el 22\% (CIDE, 1965, p. 5).

En todo el territorio nacional excepto el departamento de Montevideo, la cobertura hasta los 8 años era mucho más baja porque los niños no accedían a una educación inicial, que resultaba prácticamente inexistente fuera de las capitales e ingresaban tardíamente al primer grado de Primaria. Adicionalmente, los niños en el interior del país concluían en menor proporción la Primaria y de hacerlo continuaban en reducida proporción en la Educación Secundaria o Técnica.

Este informe sugiere que la alta cobertura reflejaba la importante valoración que la sociedad le adjudicaba a la educación, pero también el esfuerzo que desde el Estado se hacía para su provisión. En 1960 había en el país 2.162 escuelas primarias públicas y privadas, de las cuales 1316 eran públicas rurales ${ }^{6}$, y 477 estaban en localidades del interior. El número de escuelas urbanas había crecido entre 1930 y 1960 por debajo del crecimiento de la matrícula, indicando que "la relación espacial de la escuela y el medio ecológico bajo su atención se ha deteriorado" (CIDE, 1965, p. 39). Para fines de la década de los ochenta, en todas las áreas locales ("secciones") del territorio aquí estudiadas existía al menos una escuela de Educación Primaria completa.

Es precisamente el lado de la oferta educativa a nivel subdepartamental, en especial la provisión local de oportunidades de continuar la educación en el nivel medio, uno de los puntos centrales que permitieron diferenciar entre áreas locales en el trabajo de CLAEH CINAM

5 Con la excepción de los departamentos de Canelones y Colonia donde hay 3 institutos en cada uno.

6 Hasta 1967, las escuelas rurales impartían la enseñanza obligatoria en un programa de cuatro años y no de seis como en las urbanas. Esto repercute tanto en el tiempo total de socialización escolar provisto para el medio rural, en las oportunidades de aprendizaje y finalmente, en las tasas de cobertura por edad. 
(1963), y que también nos permite describir la oferta y estructura de oportunidades a fines de los ochenta. A comienzos de los años sesenta ${ }^{7}$, la existencia de una escuela rural (y de un "boliche") componía la mínima estructura (localidad de tipo F), la presencia de la Secundaria de Primer Ciclo (actualmente Media Básica) será típica de las localidades tipo C y B, en tanto que sólo en el tipo A se encontrará la Educación "Preparatoria" (en la actualidad Media Superior). De 257 localidades catalogadas por el informe, en 1963 solo en 72 existía al menos una oferta (pública o privada) de continuidad educativa finalizada la Primaria.

La Constitución de 1967 estableció la obligatoriedad del Primer Ciclo de Secundaria. Entre mediados de los sesenta y fines de los ochenta, el Estado funda 84 nuevos centros educativos, a razón de 3,8 por año. Sin embargo, ese esfuerzo institucional coexistió con la implementación del nuevo modelo económico que retaceó la inversión pública en políticas sociales. Pocos fueron los nuevos edificios construidos, y muy poco se modificó la expansión de la oferta: seis de cada diez fundaciones se concretaron en los departamentos Montevideo, del Sur y del Suroeste. Los departamentos de la frontera con Brasil son los menos favorecidos, a pesar de que durante la Dictadura hubo proyectos específicos de desarrollo para Artigas y Rivera.

Tal como se puede apreciar en la Tabla 2, en el 66\% de las áreas locales aquí definidas no hubo avances desde 1967. En el otro extremo, solo en el 5\% de las áreas locales hubo un ritmo sostenido en la ampliación de la estructura de oportunidades educativas. Además, en este panorama es muy diferente entre regiones: la región del Gran Canelones es la opuesta a las regiones Central o de la Laguna Merin.

Tabla 2. Cambio porcentual en la estructura local de oportunidades educativas 1967-1994. Creación de Liceos de Secundaria en áreas locales fuera del departamento de Montevideo según períodos (en porcentajes por región)

\begin{tabular}{l|c|c|c|c|c|c|c|c}
\hline Cambio en la Estructura & Canelones & Suroeste & Litoral & Frontera & Merin & Este & Central & Total \\
\hline Sin creación desde 1967 & 33 & 61 & 67 & 72 & 73 & 69 & 73 & 66 \\
$\begin{array}{l}\text { Con creación 1967 a 1984, } \\
\text { después no. }\end{array}$ & 29 & 13 & 9 & 8 & 13 & 6 & 13 & 12 \\
\hline $\begin{array}{l}\text { Creación entre 1985 y 1994 } \\
\text { Aumento para 1984 y para }\end{array}$ & 19 & 17 & 22 & 11 & 13 & 19 & 15 & 17 \\
1994 & 19 & 9 & 2 & 8 & 0 & 6 & 0 & 5 \\
\hline Total & 100 & 100 & 100 & 100 & 100 & 100 & 100 & 100 \\
\hline
\end{tabular}

Fuente: Elaboración propia con base en microdatos censales de 1985 y de centros educativos de la ANEP. Las fechas de fundación provienen de Bralich (2008).

7 Obsérvese que es la época en que habría nacido la mayoría de los padres de la generación de alumnos evaluados por UMRE aquí estudiada. 


\section{DATOS Y MÉTODO}

Este análisis acude a varias fuentes de información secundaria. Una parte importante del análisis territorial ha sido hecha con base en microdatos de los Censos de Población de 1963, 1975, 1985 y 1996. De estas bases se construyeron indicadores agregados para cada área local, sobre población, migración, mercado de trabajo y de escolarización.

Con base en el trabajo de Klazcko (1981) sobre los centros poblados existentes a comienzos del siglo, se integró una base de datos de localidades para 1963, 1985 y 1996 para la cual debió digitarse los índices toponímicos respectos (Davrieux, 1972; Dirección General de Estadísticas y Censos, 1989; INE, 2008) y al mismo tiempo trabajar con la cartografía para dirimir dudas relativas a la continuidad de decenas de localidades a lo largo del siglo XX.

La Dirección de Investigación, Evaluación y Estadísticas (DIEE) de la Administración Nacional de Educación Pública (ANEP) proveyó de microdatos con la localidad, la sección y el segmento censal de todos los centros educativos públicos que habían impartido Educación Inicial, Primaria y Media entre el año 2000 y el año 2017. La información sobre las escuelas primarias y jardines de infantes operativos en los años noventa fue recuperada de los Nomenclatores de Primaria de esos años.

Las variables de logro educativo y sociodemográficas utilizadas aquí provienen de los microdatos de la Evaluación Nacional de Aprendizajes en 6tos años de Educación Primaria. Esta fue realizada en octubre de 1996; tuvo carácter censal y aplicó pruebas de Lectura, Producción de Textos y Matemática a 46.641 estudiantes que tenían entre 11 y 14 años y asistían a 1.294 escuelas públicas urbanas, rurales, privadas habilitadas y privadas autorizadas en todo el territorio nacional. La distribución regional de alumnos y escuelas evaluadas se muestra en la Tabla 2.

\section{UBICACIÓN HISTÓRICA DE LA GENERACIÓN ANALIZADA}

Esta generación de alumnos, conforme a la normativa vigente en el momento de ingresar al primer grado de Primaria (años 1990 y 1991), comprende a los nacidos entre el $1^{\circ}$ de julio de 1984 y el 30 de junio de 1985. Aquí se encontraba el $70 \%$ de la generación. De haber repetido un grado o haber ingresado rezagados respecto a la edad normativa, incluiría a aquellos nacidos también los años 1983 y 1982. La distribución por años de nacimiento y edades se muestra en la Tabla 3. Conforme a esta distribución, podemos ubicar a la generación evaluada naciendo durante la etapa denominada de la transición desde la Dictadura militar a la Democracia, y comenzando la Primaria en el período que los historiadores económicos informan que explícitamente se dan pasos definitivos para implementar un nuevo modelo de base agroexportadora y de retracción del Estado. 
Tabaré Fernández, Santiago Cardozo, Mahira González y Cecilia Rodríguez

Tabla 3. Distribución de las fechas de nacimiento y edades de los niños evaluados en 1996

\begin{tabular}{l|c|c}
\hline Fechas de nacimiento (normativas) & Edades a Marzo & $\%$ \\
\hline Nacidos entre $1 / 7 / 1984$ y $30 / 6 / 1985$ & 11 & 70.8 \\
\hline Nacidos entre $1 / 7 / 1983$ y $30 / 6 / 1984$ & 12 & 20.5 \\
\hline Nacidos entre $1 / 7 / 1981$ y $30 / 6 / 1983$ & 13 y 14 & 8.7 \\
\hline Sin datos & SD & 0.1 \\
\hline Total & & 100.0 \\
\hline
\end{tabular}

Fuente: elaboración propia con base en microdatos UMRE 1996.

La información recabada sobre la madre nos permite ubicar que la mitad de ellas (54.6\%) nacieron entre 1957 y 1967, y que cuentan con un máximo de 40 años de edad. Esto las ubica creciendo durante la década del sesenta, en el contexto del estancamiento y la inflación creciente; el aumento de conflictividad sindical y movilizaciones políticas de izquierda; y cursando la Primaria en el momento del quiebre de la Democracia (1973) y la intervención militar en los Consejos de la Enseñanza Pública.

\section{VARIABLES ENDÓGENAS}

El análisis utilizará cuatro variables endógenas: i) puntaje en la prueba de Lectura de UMRE 1996; ii) puntaje en Matemática de UMRE 1996; iii) asistencia al menos a un año de la Educación Inicial; y iv) experiencia de repetición durante la Primaria. Las dos primeras ocupan el papel lógico de variable dependiente (en el sentido clásico) en tanto que las dos restantes tienen en nuestra conceptualización el papel de mediar entre la estratificación por clase y género y la adscripción territorial.

\section{VARIABLES EXÓGENAS}

Este estudio utiliza dos variables exógenas: el sexo declarado por el estudiante en el cuestionario aplicado a la Evaluación de Aprendizajes de 1996, y el índice de capital familiar global (KFG). Esta es una medida resumen de la posición del hogar estricto del estudiante en el campo social, utilizando las dos dimensiones señaladas por Bourdieu (Bourdieu, 1987) a tales efectos en su análisis de clase: la dimensión cultural y la dimensión económica. El análisis factorial fue identificado como la técnica más apropiada a utilizar dado que permitiría simultáneamente: a) someter a prueba la hipótesis de Bourdieu sobre la estructura bidimensional del campo social; y b) construir un índice sumatorio ponderado de capital familiar utilizando para ello las cargas factoriales de cada indicador social. Las variables utilizadas fueron el nivel educativo de la madre, el nivel educativo del padre, el 
número de libros en el hogar y número de ítems de confort disponible en el hogar. El análisis extrajo un único factor explicativo del $49 \%$ de la varianza y con alto valor en el test de KMO. El puntaje factorial fue reescalado entre 0 y 14 para contar con un mejor relacionamiento con los mínimos y máximos para educación y equipamiento.

Tabla 4. Estadísticos del análisis factorial, constructo de capital familiar global

\begin{tabular}{l|c|c}
\hline Variable & Factor1 & H2 \\
\hline Nivel educativo de la madre & 0.7331 & 0.5375 \\
\hline Nivel educativo del padre & 0.6888 & 0.4745 \\
\hline Equipamiento de confort en el hogar & 0.6898 & 0.4758 \\
\hline Número de libros en el hogar & 0.6765 & 0.4577 \\
\hline Eigen value & 1.9455 & \\
\hline Varianza explicada & $49 \%$ & \\
\hline KM0 test & & 0.7997 \\
\hline
\end{tabular}

Fuente: Elaboración propia con base en microdatos UMRE 1996.

\section{MÉTODO DE ESTIMACIÓN}

Para el análisis se optó por una estrategia en tres etapas. La primera fue estimar cuatro modelos multinivel (HLM) "vacíos" (sin variables explicativas) con cada una de las variables endógenas. La especificación varió tal como se muestra a continuación:

Esquema 1. Modelos de distribución de las variables endógenas y niveles de análisis a estimar por HLM

\begin{tabular}{l|c|c|c}
\hline & Distribución & Función vínculo & Niveles \\
\hline Educación Inicial & Bernoulli & Logística & 4 \\
\hline Repetición en Primaria & Bernoulli & Logística & 5 \\
\hline Puntaje en Lectura & Normal & Identidad & 5 \\
\hline Puntaje en Matemática & Normal & Identidad & 5 \\
\hline
\end{tabular}

\section{PRIMER PASO: DESCOMPOSICIÓN DE LA VARIANZA Y ESTIMACIÓN DEL PESO DEL TERRITORIO}

El primer estadístico a calcular es el coeficiente de correlación intraclase (ICC por su sigla en inglés). Este se entiende como la proporción de la varianza que el nivel de análisis de interés tiene sobre la varianza total. Para un modelo vacío de cuatro niveles con sección censal, departamento y [1], tomando varianzas [2] a todos sus términos, la participación de los niveles de análisis territoriales $(\mathrm{j}, \mathrm{k}, \mathrm{m})$, vendría dada por la ecuación [3] que denominaremos ICC territoriales (ICCT): 


$$
\begin{aligned}
& \text { [1] } y_{\mathrm{i}}=y_{000}+u_{000 \mathrm{~m}}+u_{00 \mathrm{k}}+u_{0 \mathrm{j}}+r_{\mathrm{ijkm}} \\
& \text { [2] } \operatorname{Var}\left(y_{\mathrm{i}}\right)=\operatorname{Var}\left(y_{000}\right)+\operatorname{Var}\left(u_{000 \mathrm{~m}}\right)+\operatorname{Var}\left(u_{00 \mathrm{k}}\right)+\operatorname{Var}\left(e_{0 \mathrm{j}}\right)+\operatorname{Var}\left(r_{\mathrm{ijkm}}\right) \\
& \text { [3] } I C C^{T}=\frac{\left[\operatorname{Var}\left(u_{000 \mathrm{~m}}\right)+\operatorname{Var}\left(u_{00 \mathrm{k}}\right)+\operatorname{Var}\left(u_{0 \mathrm{j}}\right)\right]}{\left(\operatorname{Var}\left(y_{000}\right)+\operatorname{Var}\left(u_{000 \mathrm{~m}}\right)+\operatorname{Var}\left(u_{00 \mathrm{k}}\right)+\operatorname{Var}\left(e_{0 \mathrm{j}}\right)+\operatorname{Var}\left(r_{\mathrm{ijkm}}\right)\right)}
\end{aligned}
$$

Las anteriores expresiones son extensibles cuando la variable dependiente es binaria y la función vínculo es una logística, aunque no existe consenso sobre cuál pueda ser la mejor representación algebraica para la varianza de nivel 1 (en este caso, individuo) (Raudenbuch \& Bryk, 2002). Aquí usamos el enfoque de Sdnidjers \& Bosker (2012). En el caso particular del ICC, este se estimará mediante la expresión:

$$
\text { [4] ICC }=\frac{\left[\operatorname{Var}\left(u_{000 \mathrm{~m}}\right)+\operatorname{Var}\left(u_{00 \mathrm{k}}\right)+\operatorname{Var}\left(u_{0 \mathrm{j}}\right)\right]}{\left(\operatorname{Var}\left(y_{000}\right)+\operatorname{Var}\left(u_{000 \mathrm{~m}}\right)+\operatorname{Var}\left(u_{00 \mathrm{k}}\right)+\operatorname{Var}\left(e_{0 \mathrm{j}}\right)+\left(\frac{\pi^{2}}{3}\right)\right.}
$$

\section{SEGUNDO PASO: ESTIMACIÓN DE LOS EFECTOS INDIVIDUALES DIRECTOS}

A continuación, se especificó para cada modelo un set de atributos individuales clásicamente trabajados (estratificación y género), pero examinando primero si hubiera una variabilidad aleatoria debida a los niveles territoriales y luego, imponiendo que aquellos tuvieran efectos fijos (no aleatorizados en los niveles superiores). Las expresiones [5] y [6] especifican el modelo logístico para el acceso a Educación Inicial y repetición, en tanto que la expresión [7] especifica el modelo para lectura. En los tres casos, son modelos donde se ha restringido la variación solo a la constante y no se han incluido regresores en los niveles superiores al individuo.

$$
\begin{aligned}
& \text { [5] } g_{\mathrm{i}}=y_{000}+y_{001} \text { Cap }+y_{002} \text { Sexo }+u_{000 \mathrm{~m}}+u_{00 \mathrm{k}}+u_{0 \mathrm{j}}+r_{\mathrm{ijkm}} \\
& \text { [6] } g_{\mathrm{i}}=y_{000}+y_{001} \operatorname{Cap}+y_{002} \operatorname{SexO}+y_{003} \operatorname{Ini}+u_{0000 \mathrm{~m}}+u_{000 \mathrm{k}}+u_{00 \mathrm{j}}+e_{0 \mathrm{~s}}+r_{\mathrm{isjkm}} \\
& \text { [7] } y_{\mathrm{i}}=y_{000}+y_{001} \operatorname{Cap}+y_{002} \operatorname{SexO}+y_{003} \operatorname{Ini}+y_{004} \operatorname{Rep}+u_{0000 \mathrm{~m}}+u_{000 \mathrm{k}}+u_{00 \mathrm{j}}+e_{0 \mathrm{~s}}+r_{\mathrm{isjkm}}
\end{aligned}
$$

\section{TERCER PASO: ESTIMACIÓN DE LOS EFECTOS VARIABLES A NIVEL DEL ÁREA LOCAL (SECCIÓN).}

Para profundizar la respuesta a la segunda pregunta, hemos de dar un paso más y estimar la expresión [9], aunque debido a restricciones numéricas de la muestra, solo se hará mediante un modelo de tres niveles que identifique fuentes de variación en el nivel escuela y en el nivel de la sección censal.

$$
\text { [9] } y_{\mathrm{i}}=y_{00}+\left(y_{01}+u_{01 \mathrm{j}}\right) \text { Cap }+y_{02} \operatorname{SexO}+y_{03} \operatorname{Ini}+y_{04} \operatorname{Rep}+u_{00 \mathrm{j}}+e_{0 \mathrm{~s}}+r_{\text {isjkm }}
$$




\section{HALLAZGOS}

\section{PESO DEL TERRITORIO}

El primer paso de las estimaciones muestra que el peso identificado para la escuela es consistente con varios antecedentes y que supera el $25 \%$ de la varianza en los aprendizajes en 6 to año, hallazgo consistente con todos los antecedentes disponibles. Novedoso es en cambio identificar que también en la repetición se identifica una heterogeneidad significativa entre escuelas que estaría en torno al 13\%, dependiendo del ejercicio. En este sentido, las estimaciones hechas no contradicen las teorías sociológicas predominantes.

Ahora bien, en cuanto al objetivo de este trabajo, resulta de interés mostrar que las estimaciones muestran una heterogeneidad estadísticamente significativa entre territorios para dos de las tres variables endógenas consideradas, aunque no para todos los niveles.

En la varianza total del acceso a la Educación Inicial, el 28,8\% estaría explicado por diferencias territoriales (HLM4), siendo aquellos atributos del área local los más relevantes, llegando a explicar una quinta parte de la heterogeneidad. Sorprende hallar que las diferencias entre departamentos son significativas $(7,9 \%)$ aunque de reducida entidad. En cambio, las diferencias entre regiones, fundadas en términos macroeconómicos, son muy reducidas.

Tabla 5. Estimación de los coeficientes de correlación intraclase para las variables intermediarias (acceso a la Educación Inicial y repetición en Primaria)

\begin{tabular}{|c|c|c|c|c|}
\hline & HLM2 & HLM3 & HLM4 & HLM5 \\
\hline \multicolumn{5}{|l|}{ Inicial } \\
\hline Suma de ICC territoriales (Sección, Departamento, Región) & $18.2 \%$ & $27.3 \%$ & $28.8 \%$ & NC \\
\hline Sección (área local) & $18.2 \%$ & $18.2 \%$ & $18.7 \%$ & NC \\
\hline Departamento & & $0.9 \%$ & $7.9 \%$ & NC \\
\hline Región & & & $2.2 \%$ & NC \\
\hline \multicolumn{5}{|l|}{ Suma de ICC escuela + territorios } \\
\hline \multicolumn{5}{|l|}{ \% del territorio en la varianza no individual } \\
\hline \multicolumn{5}{|l|}{ Repetición } \\
\hline ICC escuela & $13.1 \%$ & $13.1 \%$ & $13.1 \%$ & \\
\hline Suma de ICC territoriales (Sección, Departamento, Región) & & $0.3 \%$ & $0.8 \%$ & $0.6 \%$ \\
\hline Sección (área local) & & $0.3 \%$ & $0.5 \%$ & $0.4 \%$ \\
\hline Departamento & & & $0.3 \%$ & $0.2 \%$ \\
\hline Región & & & & $0.0 \%$ \\
\hline \multicolumn{5}{|l|}{ Suma de ICC escuela + territorios } \\
\hline$\%$ del territorio en la varianza no individual & & & & \\
\hline
\end{tabular}

Fuente: elaboración propia con base en los microdatos UMRE 1996. 
Respecto de la repetición en Primaria, el peso de lo territorial es estadísticamente significativo, pero sustantivamente despreciable. En su conjunto, los niveles no aportan más que un $0,6 \%$ en la explicación de la varianza (HLM5). En particular, solo la sección (área local) parecería tener una mínima importancia en este tema, en tanto que ni el Departamento ni la Región incidirían. La repetición resulta, por lo tanto, variable entre escuelas, con escasa correlación territorial.

Para finalizar, el peso del territorio se incrementa en la prueba de Lectura desde el primer ejercicio (HLM 3), que solo incorpora el área local hasta el último ajuste en que finalmente se agrega la región (HLM5), mostrando una vez más que el primer nivel territorial es el que presenta el mayor peso. La región, en cambio, tiene un peso ínfimo. Cuando se repite el ejercicio con la prueba de Matemática, los resultados muestran un mayor peso de la escuela en desmedro de lo territorial, aunque se mantiene el patrón de mayor peso en el área local frente a lo departamental.

Tabla 6. Estimación de los coeficientes de correlación intraclase, escuela y territoriales, para las pruebas de Lectura y Matemática

\begin{tabular}{|c|c|c|c|c|}
\hline & HLM2 & HLM3 & HLM4 & HLM5 \\
\hline \multicolumn{5}{|l|}{ Lectura } \\
\hline ICC escuela & $27.9 \%$ & $28.3 \%$ & $28.0 \%$ & $28.1 \%$ \\
\hline $\begin{array}{l}\text { Suma de ICCs territoriales (Sección, } \\
\text { Departamento, Región) }\end{array}$ & & $1.7 \%$ & $5.3 \%$ & $6.2 \%$ \\
\hline Sección (área local) & & $1.7 \%$ & $2.9 \%$ & $3.2 \%$ \\
\hline Departamento & & & $2.3 \%$ & $2.3 \%$ \\
\hline Región & & & & $0.7 \%$ \\
\hline Suma de ICC escuela + territorios & 27.9 & 31.7 & 38.5 & 40.5 \\
\hline$\%$ del territorio en la varianza no individual & $0.0 \%$ & $5.4 \%$ & $13.8 \%$ & $15.3 \%$ \\
\hline \multicolumn{5}{|l|}{ Matemática } \\
\hline ICC escuela & $31.6 \%$ & $31.7 \%$ & $31.9 \%$ & $29.1 \%$ \\
\hline $\begin{array}{l}\text { Suma de ICC territoriales (Sección, } \\
\text { Departamento, Región) }\end{array}$ & & $1.2 \%$ & $3.9 \%$ & $2.9 \%$ \\
\hline Sección (área local) & & $1.2 \%$ & $2.7 \%$ & $1.7 \%$ \\
\hline Departamento & & & $1.2 \%$ & $1.2 \%$ \\
\hline Región & & & & $\begin{array}{c}\text { Menos del } \\
1 / 00\end{array}$ \\
\hline Suma de ICC escuela + territorios & 31.6 & 34.1 & 39.7 & 34.9 \\
\hline$\%$ del territorio en la varianza no individual & $0.0 \%$ & $3.5 \%$ & $9.8 \%$ & $8.3 \%$ \\
\hline
\end{tabular}

Fuente: elaboración propia con base en los microdatos UMRE 1996. 
Entre los tres estimados, la agregación del nivel local constituye el más importante para explicar la correlación espacial. Esto figuraba como una hipótesis en el marco teórico. Pero la incidencia territorial sobre la repetición y en Matemática es bastante más débil, aunque no despreciable. Aún con estas limitaciones, es de notar que el territorio representa una séptima parte de la varianza no individual en dos de los cuatro logros educativos evaluados.

\section{SEGUNDO PASO: DETERMINANTES INDIVIDUALES}

Este paso siguió la especificación enunciada en la sección metodológica, excepto para la repetición donde optamos por no considerar la región como un nivel de análisis sino como una variable (dado que como término aleatorio no resultó significativo). Las Tablas 5, y n⿳06 muestran los resultados.

En forma consistente con los antecedentes, también es explicable la asistencia a la Educación Inicial a fines de los años ochenta conforme al volumen total de las distintas especies de capital que una familia disponía. En cambio, ha sido menos reportado y mucho menos considerado, el hallazgo de que las chances de haber cursado diferían significativamente en favor de las niñas. La diferencia es leve: en promedio, una niña aumenta en $2,8 \%$ su probabilidad de cursar. Sus implicancias hacia la trayectoria escolar, empero, no deberían ser soslayadas. La magnitud de la relación es de entidad: el coeficiente de reducción proporcional del error, o Pseudo $\mathrm{R}^{2}$ de McFadden, muestra un valor de 0,056 (Tabla 7).

Tabla 7. Coeficientes logísticos multinivel estimados para el cursado de Educación Inicial en la generación nacida entre 1981 y 1985

\begin{tabular}{l|c|c|c|c|c|c}
\hline Educación Inicial & Coeficiente & Std. Err. & $\mathbf{Z}$ & $\mathbf{P > \mathbf { Z }}$ & [95\% Conf. & Interval] \\
\hline Es mujer (en lugar de varón) & 0.1780 & 0.0310 & 5.74 & 0.00 & 0.1172 & 0.2387 \\
\hline Capital familiar global & 0.2432 & 0.0072 & 33.63 & 0.00 & 0.2291 & 0.2574 \\
\hline Constante & 0.0631 & 0.1348 & 0.47 & 0.64 & -0.2011 & 0.3273 \\
\hline
\end{tabular}

Fuente: elaboración propia con base en los microdatos UMRE 1996.

La repetición en Primaria, conforme al modelo estimado y presentado en la Tabla 8, está asociada al capital familiar y al sexo del alumno en forma altamente significativa. Si bien lo primero es consistente con antecedentes e hipótesis fundados en la teoría de la reproducción, la brecha de sexo resulta inesperada. El fracaso de los varones además resulta mayor cuanto menor es el capital familiar en el hogar. Mientras que la brecha es de $0,3 \%$ con el más alto capital, aumenta al $10,8 \%$ en los hogares totalmente carenciados. 
Más relevante a nuestra primera hipótesis, la repetición en Primaria no está asociada al contraste entre regiones del país, excepto con la Frontera Noreste con Brasil. En comparación con el Gran Canelones, la repetición es menos probable en aquella, hallazgo que no parecería ser consistente con lo que se ha descripto sobre las políticas lingüísticas nacionalistas implantadas en el magisterio durante la Dictadura (Barrios, 2001) ${ }^{8}$. En segundo lugar, y estimadas las probabilidades de repetir y de no repetir, las diferencias son leves, pero significativamente mayores cuanto menor es el capital familiar. Si bien no encontramos explicaciones en la bibliografía al porqué del primer hallazgo, está claro que el segundo sí tiene relación con una economía política de la lengua (Bourdieu, 1985).

El análisis también aporta evidencia a la segunda hipótesis general. El aprovechamiento de la oportunidad de cursar Educación Inicial tiene impacto significativo y de magnitud en la repetición. Sobre las situaciones estimadas arriba, el cursado disminuye aún más la probabilidad de repetición, sobre todo entre los niños originarios de hogares más carenciados. Dicho de otra forma, la Educación Inicial ya social y geográficamente estratificada impacta multiplicando la desigualdad de oportunidades de aprendizaje.

Tabla 8. Coeficientes logísticos multinivel estimados para la repetición en Primaria en la generación nacida entre 1981 y 1985

\begin{tabular}{l|c|c|c|c|c|c}
\hline Variables & Coef. & $\begin{array}{c}\text { Error } \\
\text { Estand }\end{array}$ & Z & \multicolumn{2}{|c}{ P>z } & \multicolumn{2}{|c}{$95 \%$ confianza } \\
\hline Es mujer en lugar de varón & -0.4706 & 0.0285 & -16.5 & 0.000 & -0.5265 & -0.4147 \\
\hline Capital familiar & -0.2870 & 0.00705 & -43.76 & 0.000 & -0.3222 & -0.2946 \\
\hline Cursó Educación Inicial & -0.5927 & 0.0343 & -17.29 & 0.000 & -0.6599 & -0.5255 \\
\hline $\begin{array}{l}\text { Vive en la región (en lugar } \\
\text { de Canelones) }\end{array}$ & & & & & \\
\hline Sur-suroeste & -0.1296 & 0.1422 & -0.91 & 0.3620 & -0.4308 & 0.1530 \\
\hline Litoral & -0.1687 & 0.1314 & -1.28 & 0.1990 & -0.4051 & 0.1345 \\
\hline Frontera Noreste & -0.3471 & 0.1351 & -2.57 & 0.0100 & -0.5603 & -0.0060 \\
\hline Cuenca de la Laguna Merin & -0.1052 & 0.1736 & -0.61 & 0.5440 & -0.4209 & 0.2854 \\
\hline Este & -0.034 & 0.1546 & -0.22 & 0.8260 & -0.3219 & 0.3082 \\
\hline Central & -0.1277 & 0.1462 & -0.91 & 0.3610 & -0.4060 & 0.1671 \\
\hline Constante & 1.2686 & 0.1205 & 10.52 & 0.000 & 1.0323 & 1.5048 \\
\hline
\end{tabular}

Fuente: Elaboración propia con base en los microdatos UMRE 1996.

8 Cabría haber hallado que la repetición hubiera aumentado, puesto que a las dificultades propias de la adquisición de la lectoescritura se agregó ese entorno de políticas que presionaba por una mayor pureza en la lengua usada. 
El último ejercicio consistió en estimar los efectos directos del capital familiar y del sexo, conjuntamente con las dos variables endógenas, cursado de nivel inicial y repetición, sobre el nivel de aprendizaje en Lectura y Matemática logrado concluyendo el sexto grado de Primaria. Las Tablas 9 y 10 presentan esta información. Una vez más, los hallazgos son altamente consistentes con los reportados en otros trabajos antes citados, incluso en lo que respecta a las diferencias observadas entre los determinantes para las dos pruebas.

El capital familiar incide levemente con mayor fuerza en Lectura que en Matemática. La magnitud de esta incidencia, si se compara el máximo y el mínimo de capital equivale a la quinta parte del puntaje de la prueba. A estos efectos directos sobre la desigualdad, deben añadirse los indirectos del capital que ya fueron estimados sobre el acceso a la Educación Inicial y sobre la repetición. Es más, este modelo identifica efectos directos de estos dos logros educativos previos. Es de notar lo que se ha dicho en varios lugares anteriores que la experiencia de reprobación, corregida a través del mecanismo de repetir el grado, está asociado a un desempeño menor en un grado significativo al completar la Primaria. Dado que no se cuenta con una medida al ingreso de Primaria que permita conocer el impacto neto de la repetición, resulta arriesgado inferir si causalmente tiene un impacto positivo o negativo. Más allá de esto, la acumulación de desigualdades por esta vía resulta evidente.

Tabla 9. Determinantes del aprendizaje en Lectura, 6to grado Primaria. Uruguay 1996

\begin{tabular}{l|c|c|c|c|c|c}
\hline Variables & Coef. & Error Estand & $\mathbf{Z}$ & \multicolumn{1}{|c|}{ P>z } & \multicolumn{2}{|c}{ 95\% confianza } \\
\hline Es mujer & 0.7893 & 0.0475 & 16.63 & 0.000 & 0.6962 & 0.8823 \\
\hline Capital familiar & 0.3197 & 0.0112 & 28.42 & 0.000 & 0.2977 & 0.3418 \\
\hline Cursó Educación Inicial & 0.3533 & 0.0634 & 5.57 & 0.000 & 0.2290 & 0.4775 \\
\hline $\begin{array}{l}\text { Repitió algún año en } \\
\text { Primaria }\end{array}$ & -1.7401 & 0.0559 & -31.14 & 0.000 & -1.8497 & -1.6306 \\
\hline $\begin{array}{l}\text { Vive en la región (en } \\
\text { lugar de Canelones) }\end{array}$ & & & & & & \\
\hline Sur-suroeste & -0.2003 & 0.4995 & -0.4 & 0.688 & -1.1792 & 0.7787 \\
\hline Litoral & -0.0961 & 0.4584 & -0.21 & 0.834 & -0.9946 & 0.8024 \\
\hline Frontera Noreste & -1.1147 & 0.4746 & -2.35 & 0.019 & -2.0449 & -0.1846 \\
\hline Cuenca de la Laguna & -0.4766 & 0.6124 & -0.78 & 0.436 & -1.6769 & 0.7236 \\
Merin & & & & & & \\
\hline Este & -0.0567 & 0.5409 & -0.1 & 0.917 & -1.1167 & 1.0034 \\
\hline Central & -0.7705 & 0.4830 & -1.6 & 0.111 & -1.7171 & 0.1761 \\
\hline Constante & 12.2126 & 0.4021 & 30.37 & 0.000 & 11.4244 & 13.0008 \\
\hline
\end{tabular}

Fuente: Elaboración propia con base en los microdatos UMRE 1996 
La estimación identificó efectos diferenciales del territorio en Lectura, pero no en Matemática. La residencia en un área local de la región de la Frontera Noreste tiene un efecto significativo y negativo sobre el logro. En términos de órdenes de magnitud absoluta, este efecto es tres veces mayor al identificado para la asistencia en la Educación Inicial. Lo que implica que un niño residente en esta región, que tiene menor probabilidad de haber cursado este nivel, agrega otra desigualdad de origen regional al cabo de la Primaria.

Globalmente, el modelo estimado para Matemática muestra menor magnitud en la relación de los determinantes con el logro (entre el $5 \%$ y el $21 \%$ menor). En el caso de Matemática, además, no habría una brecha de género ni tampoco efectos de la región de residencia. Esto debe ser analizado en conjunto con el menor ICC que se reportó más arriba para el territorio y la escuela.

Evaluados los modelos desde el punto de vista del ajuste, pueden catalogarse de forma satisfactoria. Conforme a la reducción proporcional del error, las magnitudes halladas son más bien modestas frente a las pretensiones explicativas de la teoría de Bourdieu: un 9,1\% en el nivel 1 (individuos) para la prueba de Lectura y un 7,2\% para la prueba de Matemática. Sin embargo, estas magnitudes suelen ser regularmente reportadas para este tipo de ajuste. Examinada la explicación alcanzada en el nivel 4 (departamentos) debido a la inclusión de las regiones, la magnitud fue del $68,1 \%$ para Lectura. El ICC residual en los niveles territoriales para ambos modelos varía entre $2,1 \%$ y $1,9 \%$, lo que indica que la inclusión de la regionalización socioeconómica ha contribuido a la explicación global de las diferencias entre territorios en aproximadamente la mitad de la heterogeneidad inicialmente identificada.

Tabla 10. Determinantes del aprendizaje en Matemática, 6to grado Primaria

\begin{tabular}{l|c|c|c|c|c|c}
\hline Variables & Coef. & $\begin{array}{c}\text { Error } \\
\text { Estand }\end{array}$ & Z & \multicolumn{2}{|c}{ P > } & \multicolumn{2}{|c}{$95 \%$ CI } \\
\hline Es mujer & 0.0025 & 0.0441 & 0.06 & 0.954 & -0.0839 & 0.0889 \\
\hline Capital familiar & 0.3028 & 0.0104 & 29.21 & 0.000 & 0.2825 & 0.3231 \\
\hline Cursó Educación Inicial & 0.2783 & 0.0589 & 4.73 & 0.000 & 0.1629 & 0.3937 \\
\hline Repitió algún año en Primaria & -1.4554 & 0.0515 & -28.24 & 0.000 & -1.5564 & -1.3544 \\
\hline Vive en la región (en lugar de & & & & & & \\
Canelones) & & & & & & \\
\hline Sur-suroeste & 0.4097 & 0.4749 & 0.86 & 0.388 & -0.5211 & 1.3406 \\
\hline Litoral & 0.0151 & 0.4322 & 0.04 & 0.972 & -0.8319 & 0.8622 \\
\hline Frontera Noreste & -0.2589 & 0.4514 & -0.57 & 0.566 & -1.1436 & 0.6258 \\
\hline
\end{tabular}




\begin{tabular}{l|c|c|c|c|c|c}
\hline Cuenca de la Laguna Merin & -0.1398 & 0.6008 & -0.23 & 0.816 & -1.3173 & 1.0377 \\
\hline Este & 0.2698 & 0.5243 & 0.51 & 0.607 & -0.7578 & 1.2974 \\
\hline Central & -0.3974 & 0.4656 & -0.85 & 0.393 & -1.3100 & 0.5152 \\
\hline Constante & 9.8027 & 0.3722 & 26.34 & 0.000 & 9.0732 & 10.5321 \\
\hline
\end{tabular}

Fuente: Elaboración propia con base en los microdatos UMRE 1996.

\section{TERCER PASO: PRUEBA DE HIPÓTESIS DE EFECTOS INDIVIDUALES VA- RIABLES DENTRO DE LAS ÁREAS LOCALES}

Conforme a la hipótesis propuesta, el último ejercicio consistió en ajustar modelos multinivel donde se levantara la restricción de que fueran fijos los efectos de la pendiente del capital familiar. Las Tablas 11 y 12 informan que las pruebas permiten rechazar la hipótesis nula en ambos casos.

Tabla 11. Estimación de los parámetros aleatorios, incluido el efecto del capital, modelo de Lectura con tres niveles

\begin{tabular}{|c|c|c|c|c|}
\hline Random-effects Parameters & Estimate & Std. Err. & [95\% Conf. & Interval] \\
\hline \multicolumn{5}{|l|}{ Area Local: Independent } \\
\hline $\operatorname{var(capital)}$ & 0.0035 & 0.0020 & 0.0011 & 0.0105 \\
\hline var(_cons) & 0.1807 & 0.1246 & 0.0468 & 0.6979 \\
\hline \multicolumn{5}{|l|}{ Escuela: Identity } \\
\hline var(_cons) & 4.0440 & 0.2515 & 3.5800 & 4.5681 \\
\hline Varianza (Residual, individuos) & 14.6702 & 0.1281 & 14.4214 & 14.9234 \\
\hline
\end{tabular}

Fuente: Elaboración propia con base en los microdatos UMRE 1996.

Sustantivamente, esta especificación hace razonable la hipótesis de que una de las formas en que podría haber incidido la estructura local de oportunidades, conforme a sus atributos (aquí no indicados), era modificando los efectos del capital sobre los aprendizajes. La variación en el parámetro estimado es modesta, pero indica que algunas áreas locales exacerban los efectos reproductivos de la socialización, en tanto que en otras áreas locales, estos efectos se atenúan.

Tabla 12. Estimación de los parámetros aleatorios, incluido el efecto del capital familiar, modelo de Matemática con tres niveles

\begin{tabular}{|c|c|c|c|c|}
\hline Random-effects Parameters & Estimate & Std. Err. & [95\% Conf. & Interval] \\
\hline \multicolumn{5}{|l|}{ Area Local: Independent } \\
\hline var(capital) & 0.0113 & 0.0035 & 0.0062 & 0.0207 \\
\hline $\operatorname{var}\left(\_\right.$cons) & 0.0970 & 0.1189 & 0.0088 & 1.0715 \\
\hline \multicolumn{5}{|l|}{ Escuela: Identity } \\
\hline $\operatorname{var}\left(\_c o n s\right)$ & 4.5405 & 0.2677 & 4.0450 & 5.0966 \\
\hline Varianza (Residual, individuos) & 12.8113 & 0.1110 & 12.5955 & 13.0307 \\
\hline
\end{tabular}

Fuente: Elaboración propia con base en los microdatos UMRE 1996. 


\section{DISCUSIÓN DE HIPÓTESIS Y CONCLUSIONES}

Las dos preguntas que organizaron este trabajo eran: ¿acceden los alumnos a punto de egresar de Primaria a los mismos conocimientos básicos en Matemática y Lectura independientemente de su lugar de residencia? En segundo lugar, ¿ existen variaciones territoriales en los logros sucesivos de la trayectoria escolar debidos al territorio? En ambos casos, la respuesta es afirmativa. Hemos estimado que, aunque en forma modesta, la heterogeneidad territorial equivalía entre un $4 \%$ y un 5\% a la varianza en los aprendizajes de Matemática y Lectura en 6tos grados de Primaria, medidos en 1996. Esto no quita que entre el $28,1 \%$ y el $31,9 \%$ de la varianza depende de las características de la escuela. En conjunto, el espacio de los atributos ecológicos sobre la desigualdad de aprendizaje, esto es de la estructura local de oportunidades y de la escuela en particular, tenían al final del largo período "batllista" una importancia fundamental.

Estos hallazgos, sin embargo, no permiten al menos en forma robusta sustentar la hipótesis de que tales diferencias territoriales constituyeron elementos fundamentales de la estructura de desigualdad. En primer lugar, la magnitud de las relaciones halladas en los coeficientes de correlación intraclase (ICC) no se corresponde con la esperada en los inicios. En segundo lugar, la estructura de niveles hipotetizada debió ser simplificada en el segundo paso de las estimaciones debido a que añadir el nivel región (tres términos aleatorios territoriales) no permitía converger la estimación de los determinantes. Por lo que la macroregión fue tratada como efecto fijo contextual del nivel territorial "departamento". Este hallazgo parecería aportar elementos sustantivos para al menos rechazar una versión más fuerte de la teoría de capital humano que fijaría la demanda por educación primaria con base en evaluar aspectos económicos regionales; es decir, indirectamente este trabajo permite refutar la hipótesis que habíamos esbozado en otro anterior (aunque más general) (Fernández, Rodríguez y González, 2018) .

El tercer paso en el análisis emprendido aportó evidencia, aunque si bien modesta de todos modos estadísticamente significativa, para responder a la segunda pregunta en forma más detallada. El área local también es fuente de variación en la intensidad de los efectos de la socialización familiar, al menos en los indicadores que se pueden inferir asociados a la transmisión intergeneracional del capital cultural. La heterogeneidad entre escuelas en los efectos del capital familiar había sido identificada previamente en los años noventa y también en los dos mil (Fernández, 2007a). Queda pendiente para futuros análisis establecer una descripción sobre esta nueva fuente de variación y sus posibles factores asociados. 
Todos estos hallazgos sobre la incidencia del territorio ameritan a indagar más, tanto teórica como empíricamente, en cuanto a la configuración de la estructura local de oportunidades de bienestar. En este sentido, cabría discutir, por ejemplo, si el esfuerzo y las preferencias educativas de las familias al cabo de Primaria podrían estar asociadas a la diversidad y continuidad de los niveles educativos ofertados, o si aquella demanda es más bien el resultado más específico de los atributos de la escuela y de las oportunidades de aprendizaje que ofrece de modo directo a los alumnos. ¿Cuál es el horizonte temporal en que se evalúa la utilidad de los logros escolares? Estos hallazgos parecerían apuntar que la utilidad evaluada es a corto plazo y está más relacionada con las oportunidades, costos y beneficios inmediatos existentes tanto en la localidad como en la propia escuela. En otro trabajo, y en relación a la Primaria (Fernández, 2007a), hemos mostrado que la hipótesis de la "escuela como estructura de oportunidades de aprendizaje" era más que plausible en la década del 90, y no solo en Uruguay ${ }^{9}$. Lo interesante es que la heterogeneidad en estas oportunidades próximas no ha sido explorada con relación a la heterogeneidad del territorio, algo que parecería ser muy razonable al pensar en las diferencias entre las escuelas rurales y las escuelas urbanas, por ejemplo.

Para concluir, este análisis de la desigualdad enmarcado históricamente en el cambio de modelo social entendemos que permite discutir dos conclusiones adicionales. En primer lugar, que la desigualdad educativa de base territorial estaba presente y fue más que significativa para quienes nacieron en las postrimerías del modelo "batllista". La provisión "universalista estratificada" de bienestar al decir de Filgueira y Filgueira (1994), también tuvo una dimensión territorial, hasta ahora poco estudiada. Esto es un aporte más en el diagnóstico sobre las desigualdades educativas que ha venido construyendo la academia nacional en los últimos veinte años y que permite ver la persistencia de algunos rasgos a pesar de los cambios de modelos de desarrollo económico-social.

Precisamente, una segunda conclusión general permite problematizar y abrir aún más las relaciones entre modelos de desarrollo y desigualdad educativa. ¿Cuáles han sido los efectos territoriales de la reestructura neoliberal, explícita desde 1991 pero establecida firmemente desde 1995 y vigente sin cuestionamientos hasta el 2005? No parece simple establecer hipótesis, más allá de la idea más general de que el neoliberalismo habría agudizado la desigualdad, tal como

9 La línea de investigación sobre Opportunities to Learn ha sido especialmente trabajada en el marco de las evaluaciones de aprendizaje emprendidas por el Programa Trends in Mathematics and Science (TIMSS) Study desde 1994-1995. 
reportados en los otros capítulos de este libro. Tenemos alguna evidencia de que la desigualdad ha sido persistente entre 1996 y el presente, pero este resultado promedio puede esconder comportamientos opuestos en el nivel territorial. Algunas políticas sectoriales han mejorado puntualmente la dotación de las estructuras locales de oportunidades: se han creado centros de Educación Inicial y se han abierto centros de educación media básica. Pero, por otro lado, la reducción demográfica en los niveles más pequeños de los centros de servicio (Altmann, 2016), parecería haber aumentado la brecha global entre las capitales y las pequeñas localidades. El retiro general del Estado, la práctica desaparición de la agricultura familiar (Fernández, 2002) y los cambios en el mercado de trabajo, deberían haber aumentado la heterogeneidad en la estructura local de oportunidades de bienestar y por esta vía, profundizado las desigualdades. Ahora bien, el testeo de estas hipótesis nuevas sobre cambios en el tiempo enfrenta la restricción de que luego de la Evaluación de 1996, no hubo otra de tipo censal de los aprendizajes. Se podría, sin embargo, ensayar una aproximación tentativa en continuidad con esta línea de trabajo, con la evaluación de 2013 cuya muestra resulta de importancia, aunque no censal (ANEP-DIEE, 2015). Ese será nuestra próxima tarea.

\section{BIBLIOGRAFÍA}

Aguirre, R. 2009 Las bases invisibles del bienestar. El trabajo no remunerado en Uruguay (Montevideo: Fondo de Naciones Unidas para la Mujer UNIFEM).

Altmann, L. 2016 "Pequeñas localidades en Uruguay. Evolución de datos censales 1985-2011 y aproximación al protagonismo en el Sistema Urbano Nacional" ponencia presentada en la XI Bienal del Coloquio de Transformaciones Territoriales del Comité Académico de Desarrollo Regional. Asociación de Universidades del Grupo de Montevideo. Salto: Instituto de Teoría y Urbanismo, Facultad de Arquitectura, Diseño y Urbanismo, Universidad de la República.

Alvarez Lenzi, R. 1986 Fundación de centros poblados en el Uruguay (Montevideo: Facultad de Arquitectura, Universidad de la República).

ANEP-DIEE. 2005 Panorama de la Educación en el Uruguay. Una décad de transformaciones (1992-2004) (Montevideo: Administración Nacional de Educación Pública). ANEP-DIEE. (2015). Evaluación Nacional de Aprendizajes en 6tos Primaria 2013. Montevideo: Dirección de Investigación, Evaluacion y Estadísticas, Administración Nacional de Educación Pública. 
Araújo, O. 1900 Historia de la Educación Primaria (Montevideo).

Astori, D. 1982 Neoliberalismo y crisis en la agricultura familiar uruguaya (Montevideo: Fundación de Cultura Universitaria).

Astori, D., Pérez Arrarte, C., Goyetche, L. y Alonso, J. 1982 La agricultura familiar uruguaya: origenes y situación actual (Montevideo: Fundación de Cultura Universitaria / Centro Interdisciplinario de Estudios sobre el Desarrollo Uruguay).

Barrán, J. P. y Nahum, B. 1979 Batlle, los estancieros y el Imperio Británico. Tomo I: El Uruguay del 900 (Montevideo: Banda Oriental).

Barreto, I. 2009 “Mestizas, tierras y matrimonio: los mecanismos de ascenso social de la poblacíon rural de la Banda Oriental del siglo XVIII" ponencia presentada en las X Jornadas Argentinas de Estudios de Población, San Fernando del Valle de Catamarca: Asociación de Estudios de Población de Argentina (AEPA).

Barreto, I. 2011 Estudio biodemográfico de la población de Villa de Soriano (Montevideo: CSIC, Universida de la República).

Barreto, I., Albín, E. y Barboza, M. J. 2014 Amores de frontera. Relaciones inter étnicas en la zona de Cerro Largo 1795-1811(Montevideo: Facultad de Humanidades y Ciencias de la Educación, Universidad de la República) enhttps://www. researchgate.net/publication/275584690

Barrios Pintos, A. 2009 Historia de los pueblos orientales. Tomo III: de la Guerra Grande a 1900 (Montevideo: Tradinco).

Barrios, G. 2001 "Políticas lingüísticas en el uruguay: estándares vs. Dialectos en la región fronteriza uruguayo-brasileña” en Boletim ABRALIN Vol. 24, $\mathrm{N}^{\circ} 2$ en http:/www.abralin.org/publicacao/ boletim_24.php

Barrios, G. 2014 "La denominación de variedades lingüísticas en situaciones de contacto: dialecto fronterizo, DPU, portugués uruguayo, portugués fronterizo o portuñol” en Hipperdinger Y. (ed.) Lenguas: conceptos y contactos (Bahia Blanca.: Universidad Nacional del Sur).

Becker, G. 1964 Human Capital: A Theoretical Analysis with Special Reference to Education (New York: Columbia Universidad Press).

Behares, L. 2007 "Portugués del Uruguay y Educación Fronteriza" en Brovetto, C. y Brian N. G. Portugués del Uruguay y Educación Bilingüe (Montevideo: Administración Nacional de Educación Pública / Consejo de Educación Inicial y Primaria).

Bernstein, B. 1971 Class, Codes and Control. Volume 1: Theoretical Studies Towards a. Sociology of Language (London: Routdledge).

Bértola, L. 1991 La industria manufacturera uruguaya 1913-1961. Un enfoque sectorial de su crecimiento, fluctuaciones y crisis 
(Montevideo: CIUEDUR y Facultad de Ciencias Sociales, Universidad de la República).

Blanco, E. 2011 Los límites de la escuela. Educación, desigualdad y aprendizajes en México (México, D.F.: El Colegio de México).

Blanes, A., Koolhaas, M., Nathan, M. y Pardo, I. 2018 "Las tendencias demográficas a nivel subnacional en Uruguay: ¿convergencias o divergencia?” en Simpson, L. y González, L. ¿Convergencia demográfica? Análisis comparativo de las tendencias demográficas subnacionales en América Latina ( Río de Janeiro: Asociacion Latinoamericana de Población).

Bogliaccini, J.y Rodriguez, F. 2015 "Regulación del sistema educativo y desigualdades de aprendizaje en el Uruguay" en CEPAL $\mathrm{N}^{\circ} 116$ en https://repositorio.cepal.org/handle/11362/38798

Boudon, R. 1982 La desigualdad de oportunidades (Barcelona: Laia).

Bourdieu, P. 1985 ¿Qué significa hablar? Economía de los intercambios lingüísticos (Madrid: Akal).

Bourdieu, P. 1987 "The forms of capital" en Richardson J. Handbook of Theory and Research in Sociology of Education (New York: Greenwood Press).

Bourdieu, P. Passeron, J. C. 2004 (1964). Los herederos. Los estudiantes y la cultura. (Buenos Aires: Siglo XXI).

Bralich, J. 2008 "Antecedentes y marco institucional" en Nahúm B. (ed.) Historia de la Educación Secundaria 1935-2008 (Montevideo: CES).

Braudel, F. 1958 "Histoire et sciences sociales. La longue durée" e. Annales. Économies, Sociétés, Civilisations Año 13 a, No 4.

Breen, R. y Goldthorpe, J. 1997 "Explaining educational differences. Towards a formal rational action theory" en Rationality \& Society Vol. 9, $\mathrm{N}^{\circ} 3$.

Bunge, M. 1999 Buscar la Filosofía en las Ciencias Sociales (México, D.F.: SIGLO XXI).

Cardoso, F. H. y Faleto, E. 1969 Dependencia y desarrollo en América Latina (México, D.F.: Siglo XXI).

Castro, J. 1949 "Coordinación entre Primaria y Secundaria” en CNEPyN Anales de Instrucción Primaria (Vol. tomo XII) (Montevideo: CNEPyN).

CEPAL. 1991 Enseñanza primaria y ciclo básico de educación media en el Uruguay. (Montevideo: Comisión Económica para América Latina y el Caribe (CEPAL), Naciones Unidas).

Cervini, R. y Basualdo, M. 2003 "La eficacia del sector público. El caso de las escuelas secundarias técnicas en Argentina" en Revista Latinoamericana de Estudios Educativos Vol. XXXIII, $\mathrm{N}^{\circ} 3$. 
Checchi, D. 2006 The Economics of Education. Human capital familiy background and inequality (Cambridge, UK: Cambridge University Press).

CIDE. 1965 Informe sobre el Estado de la Educación en Uruguay (Montevideo: Ministerio de Instrucción Pública y Previsión Social).

CLAEH / CINAM. 1963 Situación económica y social del Uruguay Rural (Montevideo: Centro Latino Americano de Economía Humana (CLAEH) / Ministerio de Ganadería, Presidencia de la República).

Cocchi, M. A., Klaczko, J. y Rial, J. 1977. El proceso de poblamiento de Uruguay en los siglos XVIII y XIX. Documento de Trabajo $N^{o} 20$ /Montevideo: Centro de Informaciones y Estudios Sociales del Uruguay (CIESU)).

Coleman, J. S. 2011 (1990) Fundamentos de teoría social (Madrid: Centro de Investigaciones Sociológicas).

Coraggio, J. L. 1972 "Hacia una revisión de la teoría de los polos de desarrollo" en Revista Latinoamericana de Estudios Urbanos y Regionales (EURE) Vol. II, N 4. Recuperado, de http://eure.cl/ index.php/eure/article/view/820/677 acceso el 5 de noviembre de 2018

Davrieux, A. 1972 Índice toponímico de lugares poblados del Uruguay (Montevideo: Dirección General de Estadística, Presidencia de la República).

DIEA. 2014 “El Uruguay agropecuario 1900 al 2000” en Revista del Plan Agropecuario $\mathrm{N}^{\circ} 149$ en www.planagropecuario.org.y/ publicaciones/revisa/R149/R_149_64.pdf

Dirección General de Estadísticas y Censos. 1989 Indice toponímico de entidades de población: VI censo de población, IV censo de viviendas 1985 (Montevideo: Dirección General de Estadística y Censos, Presidencia de la República).

Doyal, L. y Gough, I. 1991 A Tbeory of Human Needs (Basingstoke, UK: MacMillan).

Esping-Andersen, G. 1990 The Three Worlds of Welfare Capitalism (New Jersey: Princeton University Press).

Fernández, T. 2002 "Cambios en la estructura agraria de Uruguay entre 1951 y 2000" en Estudios Sociológicos Vol. XX, № 2.

Fernández, T. 2002 "Determinantes sociales e institucionales de la desigualdad educativa en sextos años de educación primaria de Argentina y Uruguay. Una aproximación mediante un modelo de regresión logística" en Revista Mexicana de Investigación Educativa Vol. 7, $\mathrm{N}^{\circ} 16 \mathrm{en} \mathrm{http://www.redalyc.org/}$ pdf/140/14001605.pdf 
Fernández, T. 2003 "La desigualdad educativa en Uruguay entre 1996 y 1999" en REICE - Revista Electrónica Iberoamericana sobre Calidad, Eficacia y Cambio en Educación Vol. 1, N 1.

Fernández, T. 2007a Distribución del conocimiento escolar: clases sociales, escuela y sistema educativo en América Latina (México, D.F.: El Colegio de México).

Fernández, T. 2007b "Persistent Inequalities in Uruguayan Primary Education 1996-2002" en Teese R., Lamb S. y Duru-Bellat, M. (eds.) en International Studies in Educational Inequalitiy, Theory and Policy. Volume Two: Inequality in Education Systems (Dordrecht: Spinger).

Fernández, T. 2018a La conformación de las regiones en Uruguay desde un enfoque histórico-estructural. Un ensayo de síntesis (Montevideo: Documento de Trabajo).

Fernández, T. 2018b La conformación histórica de las regiones en Uruguay (1730-1980). Un ensayo de síntesis desde la sociología histórica (Montevideo: Documento de Trabajo, Departamento de Sociología, Facultad de Ciencias Sociales, Universidad de la República).

Fernández, T.y Ríos, A. 2013 Implicancias para la política educativa de las tendencias socioeconómicas y educativas registradas en el espacio regional "Frontera-Nordeste" de Uruguay (Montevideo: Manuscrito disponible en web. Departamento de Sociología, Facultad de Ciencias Sociales; y Centro Universitario de Rivera; Universidad de la República).

Fernández, T., González, M., Marques, A.y Rodriguez, C. Diferenciación regional y desigualdades territoriales en Uruguay (1730-1970). doi:10.13140/RG.2.2.27912.93445 acceso 24 de octubre de 2018 .

Fernández, T., Rodríguez, C. y González, M. 2018 “Teoría social, regionalización histórica y mecanismos que inciden en la desigualdad educativa en Uruguay" en Mazzei E. (ed.). (Melo: Centro de Estudios sobre la Frontera, Casa de la Universidad de Cerro Largo, Universidad de la República).

Filgueira, C. y Filgueira, F. 1994 El largo adiós al país modelo. Políticas sociales y pobreza en el Uruguay (Montevideo: ARCA / Peithos Sociedad de Análisis Político / Kellogs Institut).

Giddens, A. 1992 La constitución de la sociedad. (Buenos Aires: Amorrortu).

INE. 2008 Toponimia y categorización jurídica oficial de localidades urbanas del Uruguay (Montevideo: Instituto Nacional de Estadística, Presidencia de la República).

Jacob, R. 1981 Breve Historia de la Industria en Uruguay 
(Montevideo: Fundación de Cultura Universitaria).

Kaztman, R. y Filgueira, C. 1999 Activos y estructura de oportundiades. Estudios sbore las raices de la vulnerabilidad social en Uruguay" (Montevideo: Oficina de la CEPAL).

Kaztman, R. y Retamoso, A. 2007 "Efectos de la segregación urbana sobre la educación en Montevideo" CEPAL $\mathrm{N}^{\circ} 91$ enhttps://www. cepal.org/es/publicaciones/37451-cepal-review-91

Klazcko, J. 1981 El Uruguay de 1908: su contexto urbano-rural, antecedentes y perspectivas (Montevideo: CIESU. Cuaderno $\mathrm{N}^{\circ}$ $\left.{ }^{\circ} 42\right)$.

Lazarsfeld, P. y Menzel, H. 1961 "On the relation between individual and collectives properties" en Etzioni A. (ed.) Complex organizations. A sociological reader (New York: Holt, Rinehart \& Winston Press).

Mazzei, E. yDe Souza, M. 2013 La frontera en cifras (Melo: Comisión Coordinadora del Interior / Universidad de la República).

Moraes, M. I. 2013 Mundos rurales. Nuestro tiempo Vol. 16 (Montevideo: IMPO, Presidencia de la República Oriental del Uruguay).

Oroño, M. 2016 "La escuela en la construcción de fronteras culturales y lingüisticas en el Uruguay de fines del siglo XIX" en Páginas de Educación Vol. 9, $N^{\circ} 1$ en En https://revistas.ucu.edu. uy/index.php/paginasdeeducacion/article/view/1182/1141 acceso 17 de agosto de 2017.

Paasi, A. 1986 "The institutionalizacion of regions: a theoretical framework for understanding the emergence and the constitutioin of regional identity" en FENNIA Vol. 164, N 1. doi:10.11143/9052

Paasi, A y Mezger, J. 2016 "Foregrounding the region" en Regional Studies Vol. 51, $\mathrm{N}^{\circ} 1$

Petruccelli, J. L. 1976 Notas sobre el proceso de poblacmiento uruguayo. Documento de Trabajo $N^{o} 14$ (Montevideo: Centro de Informaciones y Estudios del Uruguay (CIESU)).

Piñeiro, D. 1987 Formas de resistencia de la agricultura familiar. El caso del noreste de Canelones . (Montevideo: CIESU).

Rama, G. 1991 Qué aprenden y quiénes aprenden en las Escuelas Primarias de Uruguay (Montevideo: Oficina de la CEPAL; Naciones Unidas).

Raudenbuch, S. y Bryk, A. 2002 Hierachical Linear Models. 2 Ed. (Thousand Oaks,: SAGE).

Raudenbush, S. y Willms, D. 1995 "The estimation of school effects" en Journal of Educational and Behavioral Statistics Vol. 20, $\mathrm{N}^{\circ} 4$.

Ravela, P., Picaroni, B., Loureiro, G., Luaces, O., Gonet, D. y 
Fernández, T. 2000 Evaluaciones nacionales de aprendizaje en Educación Primaria de Uruguay (1995-1999) (Montevideo: Unidad de Medición de Resultados de Educativos (UMRE)/ Administración Nacional de Educación Pública (ANEP)).

Sen, A. 1985 Commodities and Capabilities (Amsterdam: North Holland).

Sewell, W. J. 1992 "A Theory of Structure: Duality, Agency and Transformation" en The American Journal of Sociology Vol. 98, $N^{\circ} 1$.

Sewell, W. J. 2006 "Three temporalities. Toward an Eventfull Sociology " en The Logic of History.

Shields, R. 1991 Places in the margin. Alternatives geographies of modernity (London: Routledge).

Snidjers, T. y Bosker, R. 2012 Multilevel analysis. 2 ed. (London: Sage).

Solari, A. 1954 Sociología rural nacional (Montevideo: Editorial de la Facultad de Derecho, Universidad de la República).

Travieso, E. 2017 Railroads and Regional Economies in Uruguay, c1910. Revista Uruguaya de Historia Económica, 30-47.

Tuñón, I. y Poy, S. 2016 Las múltiples dimensiones de la pobreza infantil. Incidencia, evolución y principales determinantes. Período 2010-2015 (Buenos Aires: Observatorio de la Deuda Social Argentina. Universidad Católica Argentina).

Veiga, D. 1991 Desarrollo regional en el Uruguay (Montevideo: Centro de Informaciones y Estudios del Uruguay).

Veiga, D. 2010 Estructura social y ciudades en el Uruguay (Montevideo: CSIC-UDELAR / Facultad de Ciencias Sociales-UDELAR).

Veiga, D. y Lamshtein, S. 2015 Desigualdades sociales y territoriales en Uruguay. (Montevideo: Departamento de Sociología, Facultad de Ciencias Sociales y CSIC, Universidad de la República). 